\title{
Miscibility and Rapid Phase Transition Behavior in Poly- (vinylidene fluoride-co-hexafluoro acetone)/ Poly(ethylene-co-vinyl acetate) Blends
}

\author{
Masatoshi HaSEgAwA* and Saburo AKIYAMA \\ Laboratory of Chemistry, Faculty of General Education, \\ Tokyo University of Agriculture and Technology, \\ 3-5-8 Saiwai-cho, Fuchu-shi, Tokyo 183, Japan
}

(Received November 16, 1987)

\begin{abstract}
The phase equilibrium and phase transition for miscible polymer blends of poly(vinylidene fluoride-co-hexafluoro acetone) [p(VDF-HFA)]/poly(ethylene-co-vinyl acetate) (EVAc) were investigated using DSC, dynamic mechanical loss measurements, and thermophotometry. It was found that blends of $\mathrm{p}$ (VDF-HFA) and EVAc with vinyl acetate (VAc) content more than $86.0 \mathrm{wt} \%$ are miscible, but $\mathrm{p}(\mathrm{VDF}-\mathrm{HFA}) / \mathrm{EVAc}$ with less than $45.4 \mathrm{wt} \%$ of VAc are immiscible. It was also found that a blend of p(VDF-HFA)/EVAc (VAc: $70 \mathrm{wt} \%$ ) (EVAc 70) has a LCST type phase diagram, and shows markedly rapid phase transition with temperature dropping.
\end{abstract}

KEY WORDS Blend / Miscibility / Phase Diagram / Phase Transition / Poly(vinylidene fluoride-co-hexafluoro acetone) / Poly(ethylene-co-vinyl acetate) / Activation Energy / LCST / Torsional Braid Analysis /

A considerable number of reports are available on miscible polymer blends. ${ }^{1}$ In particular, the phase diagram of polymer blends is of interest in the fields of materials science, for it makes the thermodynamical approach to polymer blends possible.

If there are attractive intermolecular interactions between polar groups in polymer blends, the system can exhibit a LCST (lower critical solution temperature) type phase diagram. As reported previously, a polar polymer blend, poly(vinyl nitrate)(PVN)/poly(methyl acrylate)(PMA) ${ }^{2}$ showed a LCST phase diagram. In addition, comparatively rapid phase transition behavior from the initial two phases to one phase (phase transition time $\left(\tau_{\mathrm{tr}}\right)$ : about ten minutes) was observed.

In this paper we report the phase equilibrium and phase transition of $\mathrm{p}$ (VDF-HFA)/
EVAc blends in which specific interactions between the polar groups are expected. Recently, Miyata and coworkers reported a LCST type phase diagram of $\mathrm{p}$ (VDF-HFA)/ poly(methyl methacrylate)(PMMA) blends. ${ }^{3}$ Furthermore, a remarkable rapid phase transition from two phases to one phase are detected in this system. These results support our consideration about the effects of hexafluoro acetone (HFA) unit on the mobility of polymer chain in the blends.

\section{EXPERIMENTAL}

A random copolymer $\mathrm{p}$ (VDF-HFA) (HFA: $8.9 \mathrm{~mol} \%$ ) was supplied by Central Glass Co., Ltd. The number- and weight-average molecular weights determined by GPC (Toyo Soda, HLC802UR, colum; GMIXH6, solvent;

* Present address: Research Center for Advanced and Technology, University of Tokyo, 4-6-1 Komaba, Meguro-ku, Tokyo 153, Japan 
tetrahydrofuran (THF)) were $\bar{M}_{n}=10.6 \times 10^{4}$, and $\bar{M}_{w}=22.6 \times 10^{4}$, respectively. The degrees of polymerization of EVAc and poly(vinyl acetate) (PVAc), which were supplied by Nippon Goseikagaku Co., Ltd. and Wako Junyaku Co., Ltd., respectively, are shown in Table I. The vinyl acetate (VAc) content of EVAc used in this work was in the range of 92.9 to $45.4 \mathrm{wt}^{\circ} \%$. The chemical structure for each component is shown in Figure 1.

The as-received polymers were purified by repeating reprecipitation twice from THF solution. Polymer blends were prepared by casting $c a .5 \mathrm{wt} \%$ solution, which were prepared by dissolving the requisite amounts of two polymers in THF at room temperature. The as-cast films were dried in vacuo at $50^{\circ} \mathrm{C}$ for a week.

The temperature dependence of dynamic mechanical loss was estimated from the logarithmic decrement measured with a handmade torsion pendulum apparatus. ${ }^{4}$ The blend samples of about $20 \mathrm{mg}$ were rapidly hot pressed at $140^{\circ} \mathrm{C}$ on the paper braids $(4 \mathrm{~mm} \times$ $55 \mathrm{~mm}$ ), and heated at a heating rate of about $1^{\circ} \mathrm{C} \mathrm{min}^{-1}$. The dynamic mechanical loss of the paper braids was not observed in all range of measuring temperature.

The DSC (Du Pont 990) was used for determining the $T_{\mathrm{g}}$ of samples at heating rates of $5,10,20,50$, and $100^{\circ} \mathrm{Cmin}^{-1}$.

The phase diagram for $p$ (VDF-HFA)/ EVAc70 blend was determined by the temperature dependence of percent transmittance of blend films in the thermo-optical method. At the same time, a theoretical curve was calculated from Flory-Huggins-Scott's lattice model. ${ }^{5,6}$

\section{RESULTS AND DISCUSSION}

The experimental results of dynamic mechanical loss behavior of the blends are shown in Figures 2 to 4 . The p(VDF-HFA)/ EVAc92.9 (VAc: $92.9 \mathrm{wt} \%$ ) blends showed single loss peaks independent of blend com-
Table I. Degree of polymerizations of used samples

\begin{tabular}{lll}
\hline \multicolumn{1}{c}{ Samples } & \multicolumn{1}{c}{$\bar{M}_{w}, D P$} & \multicolumn{1}{c}{ Company Ltd. } \\
\hline p(VDF-HFA) & $\bar{M}_{w}=225800$ & Central Glass \\
PVAc & $D P=1600$ & Wako Jyunyaku \\
EVAc 92.9 & $D P=2000$ & Nippon Goseikagaku \\
EVAc 86.0 & $D P=3000$ & Nippon Goseikagaku \\
EVAc 70.0 & $D P=5350$ & Nippon Goseikagaku \\
EVAc 56.1 & $D P=1950$ & Nippon Goseikagaku \\
EVAc 45.4 & $D P=2000$ & Nippon Goseikagaku \\
\hline
\end{tabular}

Component 1<smiles>FC(F)(F)C(F)(F)C(F)(F)C(F)(F)C1CC1</smiles>

Poly(vinylidene fluoride-co-hexafluoro acetone)

$\mathrm{P}(\mathrm{VDF}-\mathrm{HFA}) \quad \overline{\mathrm{Mn}}=106000$

$\overline{\mathrm{MW}}=226000$

Component 2

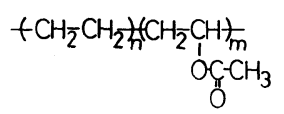

Poly(ethylene-co-vinylacetate) EVAC

Figure 1. Chemical structures of blend components.

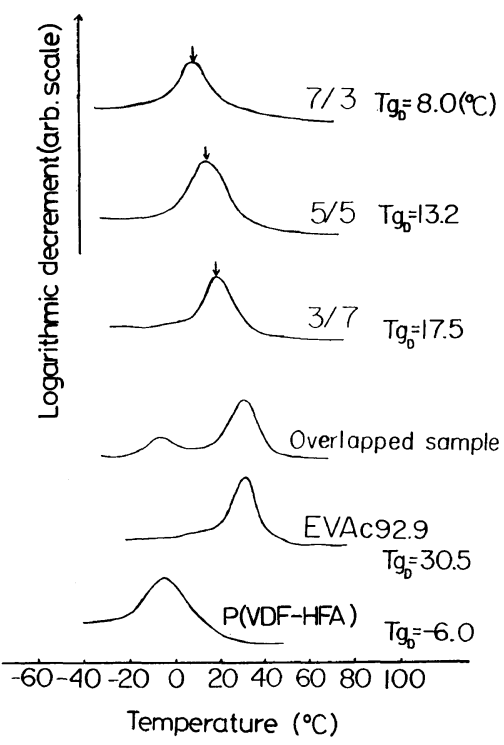

Figure 2. Dynamic mechanical loss of the $\mathrm{p}(\mathrm{VDF}$ HFA)/EVAc92.9 blends and overlapping sample with each homopolymer. 


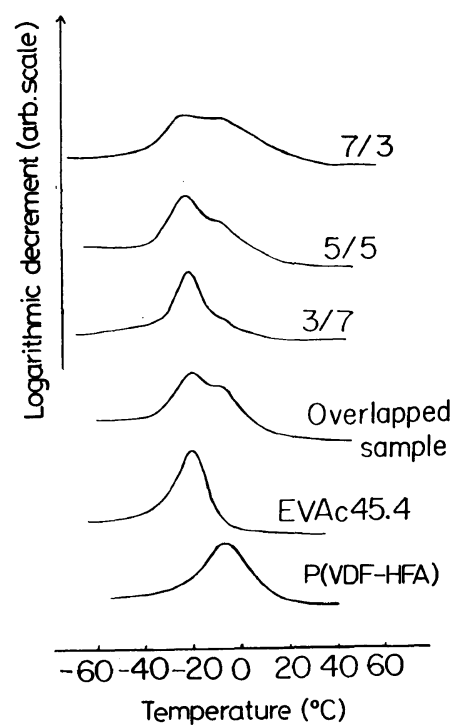

Figure 3. Dynamic mechanical loss of the $p(V D F-$ HFA)/EVAc45.4 blends and overlapping sample with each homopolymer.

positions (p(VDF-HFA)/EVAc92.9 $=7 / 3,5 / 5$, $3 / 7)$ at temperatures between the peaks of two component homopolymers. On the contrary, the overlapping samples in which the two homopolymers were hot pressed individually on the front and back side of the paper braids exhibited double peaks, as expected for the case of no-mixing. Thus the result in Figure 2 are considered to show that these blends are miscible. On the other hand, the blend with $45.4 \mathrm{wt} \%$ VAc in EVAc represented double peaks corresponding to each peak of component polymers as shown in Figure 3. This blend system is considered immiscible, based on these results. In the case of Figure 4, however, it was difficult to evaluate the miscibility of blends because of their Tgs very close to each other. All results of blends with EVAc having various VAc contents are shown in Table II. In the case of EVAc with high VAc content $\left(86.0,92.9,100 \mathrm{wt}^{\mathrm{o}} \%\right)$, the blends should be miscible in whole blend ratio at the temperature in the experiments, while the phase separation was observed at less than $45.4 \mathrm{wt} \%$ of VAc content. From these results,

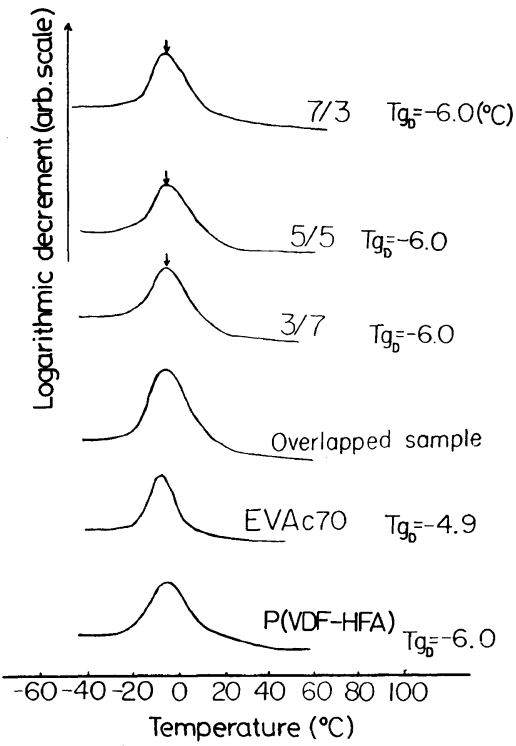

Figure 4. Dynamic mechanical loss of the $p(V D F-$ HFA)/EVAc70 blends and overlapping sample with each homopolymer.

Table II. Predicted miscibility from TBA measurements

\begin{tabular}{ccl} 
Component 1 & Component 2 & Possible blend state \\
\hline & PVAc & Completely miscible \\
& EVAc 92.9 & Completely miscible \\
p(VDF-HFA) & EVAc 86.0 & Completely miscible \\
& EVAc 70.0 & Semi-miscible \\
& EVAc 56.1 & Semi-miscible \\
& EVAc 45.4 & Immiscible \\
\hline
\end{tabular}

we consider that the specific intermolecular interactions between the polar groups (acetyl and fluoride groups) contribute predominantly to compatibility in the blend system.

Although miscibility could not be evaluated for the $p$ (VDF-HFA)/EVAc70 blend by dynamic mechnical loss measurements, this system was predicted as a semi-miscible system because these blend films changed from transparent to opaque by temperature jumping. The temperature dependence of percent transmittance by thermo-photometry at a heating rate of about $1^{\circ} \mathrm{C} \mathrm{min}^{-1}$ is shown in Figure 5. The phase diagram determined by cloud points provides a LCST type as shown in Figure 6. At 


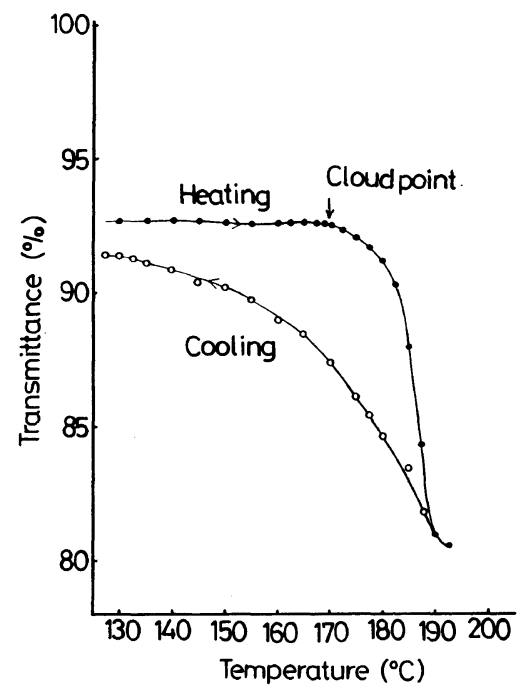

Figure 5. Thermo-photometry for $p$ (VDF-HFA)/ EVAc70 blends (5/5).

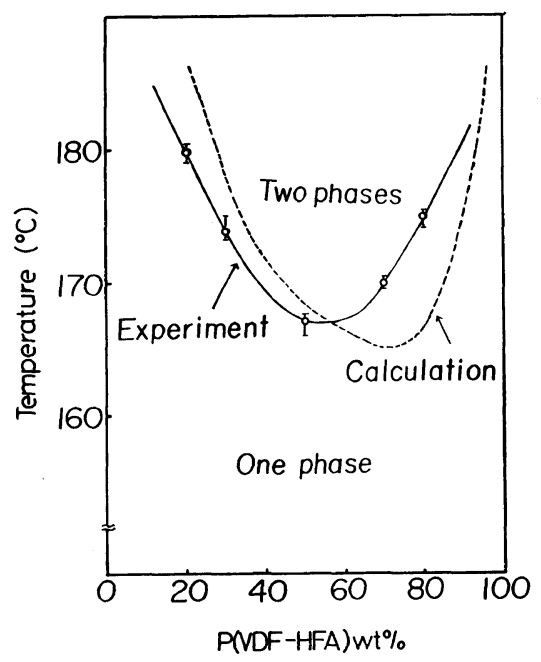

Figure 6. Phase diagram of $p($ VDF-HFA)/EVAc70 blends. Experimental (-); calculation (------).

the same time, the calculation curve from phase equilibrium, given by Newton's method, ${ }^{1}$ is also plotted. The $\chi$ parameter used for the calculation can be generally written as follows.

$$
\chi_{12}=A+B / R T
$$

where suffixes 1, 2 show $\mathrm{p}$ (VDF-HFA) and EVAc70, respectively. The parameters used are $D P_{1}$ (degree of polymerization $)=3070$,

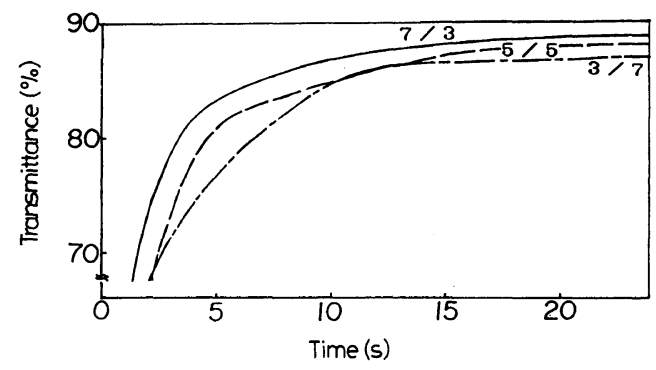

Figure 7. The rapid phase transition for the $p(V D F-$ HFA)/EVAc70 blends $(7 / 3,5 / 5,3 / 7)$ after rapidly cooling from $200^{\circ} \mathrm{C}$ to $25^{\circ} \mathrm{C}$.

$D P_{2}=5350, d_{1}$ (density) $=1.69 \mathrm{~g} \mathrm{~cm}^{-3}, d_{2}=$ $1.13 \mathrm{~g} \mathrm{~cm}^{-3}, A=4.5 \times 10^{-3}, T_{\mathrm{c}^{\infty}}=-B / A R=$ $390 \mathrm{~K}$, where $T_{\mathrm{c}^{\infty}}$ is the critical temperature of the blend having the infinite degree of polymerization of both components, and $R$ is gas constant. In eq. 1 , the first term $A$ is entropic term and positive as usual. It is therefore unfavorable for miscibility. The second term $B / R T$ is an enthalpic term. When $B$ is negative, the second term contributes mainly to miscibility at low temperature. This consideration is consistent with the experimental results in which transparent films were found at all compositions at $25^{\circ} \mathrm{C}$ and $-20^{\circ} \mathrm{C}$. The deviation between experimental and theoretical curves may be due to the disregard of molecular weight distribution in the latter.

Furthermore, this $p$ (VDF-HFA)/EVAc70 blend displayed a markedly rapid phase transition from two phases to one phase by quenching in the LCST phase diagram. Figure 7 shows the time dependence of the transmitted light intensity. By quenching from $200^{\circ} \mathrm{C}$ to $25^{\circ} \mathrm{C}$, the blend films changed from cloudy to clear for about $15 \mathrm{~s}$ as shown in figure. No fixation of the phase separation by quenching was indicated in this blend. It was found that the phase transition is extremely rapid for this system even if one compares with that of the PVN/PMA system. ${ }^{2,11}$ In most of the phase diagram systems, the reversible phase transition behavior is not exhibited by quenching from high temperature to r.t. because of the 
lack of mobility in polymer chains at r.t. This phenomenon is due to the fact that the segmental motion of polymer chains is not frozen at $25^{\circ} \mathrm{C}$, because the $T_{\mathrm{g}}$ of blend exists below r.t.

Recently, Miyata and coworkers ${ }^{3}$ reported that the $\mathrm{p}$ (VDF-HFA)/PMMA blend shows a very fast phase transition, whereas $\mathrm{PVDF} /$ PMMA $^{7}$ and PVDF/EVAc blends ${ }^{8}$ do not show such reversible and rapid behavior. Consequently we consider that hexafluoro acetone (HFA) unit should promote conformational change of polymer chains for reptation motion in the blend.

In addition, the $p$ (VDF-HFA)/EVAc70 as well as $\mathrm{p}$ (VDF-HFA)/PMMA system showed the partial formation of "blue film" at the intermediate state from two phases to one phase. This is attributed to Rayleigh scattering at "sea-island" phase separation surfaces.

In order to obtain indication of the chain mobility in polymer blends, the apparent activation energies $\left(E_{\mathrm{a}}\right)$ of glass transition process for blends were obtained by DSC method. Barton et al. ${ }^{9}$ used the following equation to get the apparent activation energies of segmental motion for homopolymers.

$$
\ln \left(v / T_{\mathrm{g}}^{2}\right)=C-E_{\mathrm{a}} / R T_{\mathrm{g}}
$$

where $C$ is constant for polymer used, $T_{\mathrm{g}}(\mathrm{K})$ is the glass transition temperature at all linear heating rates $v$. The slopes of $\ln \left(v / T_{\mathrm{g}}{ }^{2}\right)$ versus $1 /$ $T_{\mathrm{g}}$ plots provide $E_{\mathrm{a}}$, which reflects the mobility of polymer chains at $T_{\mathrm{g}}$. This equation can be applied to miscible polymer blends. Figure 8 shows the heating rate dependence of $T_{\mathrm{g}}$ in the blends. Substituting these data into eq $2, E_{\mathrm{a}}$ was obtained from the slope of straight line as seen in Figure 9. The values of $E_{\mathrm{a}}$ obtained seemed reasonable for the segmental motions of vinyl polymers. For examples, values of 40 to $80 \mathrm{kcal} \mathrm{mol}^{-1}$ were obtained for PVN/poly(vinyl acetate) (PVAc) system ${ }^{10}$ by dielectric relaxation, and also of 13 to $59 \mathrm{kcal} \mathrm{mol}^{-1}$ for PVN/PMA system ${ }^{11,12}$ by dynamic mechanical loss measurements. The

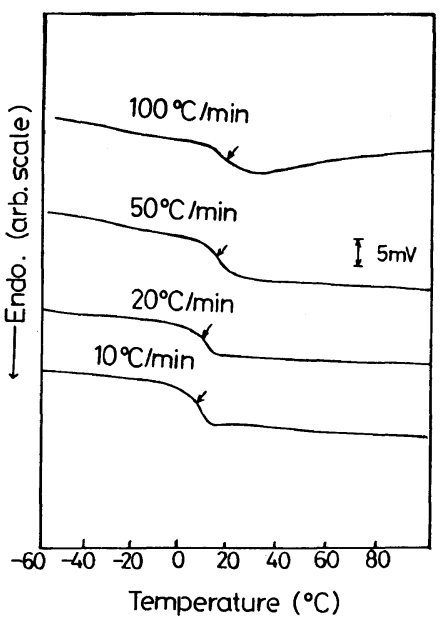

Figure 8. DSC curves of the p(VDF-HFA)/EVAc92.9 blend $(5 / 5)$ at various heating rates.

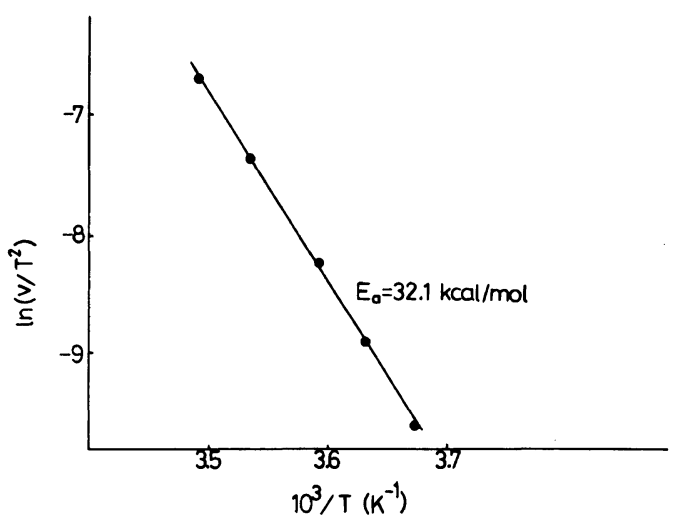

Figure 9. The plot of $\ln \left(v / T_{\mathrm{g}}{ }^{2}\right) v s .1 / T_{\mathrm{g}}$ for the $\mathrm{p}$ (VDFHFA)/EVAc92.9 blend (5/5). $T_{\mathrm{g}}$; glass transition temperature $(\mathrm{K})$.

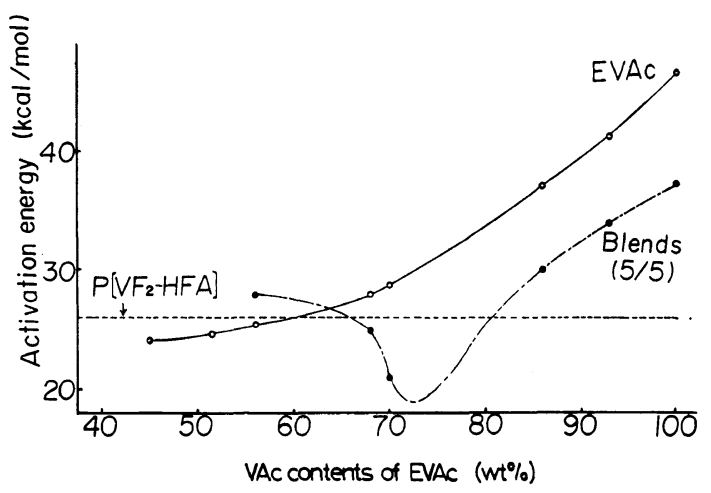

Figure 10. Activation energies of segmental motions for each component and $p($ VDF-HFA)/EVAc (5/5) against VAc content. 
values of $E_{\mathrm{a}}$ at various VAc content are shown in Figure 10 for the blend ratio 5/5. As can be seen, p(VDF-HFA)/EVAc (VAc: 86.0, 92.9, $100 \mathrm{wt} \%$ ) has a intermediate value of $E_{\mathrm{a}}$ between those of component polymers. It is noteworthy that the $E_{\mathrm{a}}$ values of blends with EVAc (VAc: $68.0,70.0 \mathrm{wt} \%$ ) are lower than those of component polymers. In the case of the $\mathrm{p}$ (VDF-HFA)/EVAc (VAc: $56.1 \mathrm{wt} \%$ ) blend with a LCST, no reversible phase transition was recognized even by the same quenching. This may be due to higher $E_{\mathrm{a}}$ of the latter blend (Figure 10).

Thus the mobility of polymer main chains in the miscible blends seems to play a very important role in the reversible and fast phase transition behavior.

\section{CONCLUSIONS}

It was found that the $\mathrm{p}$ (VDF-HFA)/ EVAc70 blend exhibited a LCST phase diagram. The phase transition in this system occurred very rapidly (completed within about $15 \mathrm{~s}$ ) on quenching the blends from $200^{\circ} \mathrm{C}$ to $25^{\circ} \mathrm{C}$. The formation of a "blue film" was also recognized for these blends at the intermediate stage of the phase transition.

This blend showed a lower apparent activation energy for segmental motions than that for the each component homopolymer.

The miscibility of $p$ (VDF-HFA)/EVAc sys- tem could be controlled by the VAc content of EVAc.

Acknowledgment. We are grateful for the helpful discussion with Prof. R. Kaneko, and Prof. S. Miyata of Tokyo University of Agriculture and Technology. Gratitude is also extended to Dr. K. Maeda of Central Glass Co., Ltd. and Dr. T. Iwanami of Nippon Goseikagaku Co., Ltd. for providing the $\mathrm{p}$ (VDF-HFA) and EVAc, respectively, and Prof. I. Mita and Prof. K. Horie of University of Tokyo for making the GPC measurements.

\section{REFERENCES}

1. S. Akiyama, T. Inoue, and T. Nishi, "Polymer Blends," CMC Press, Tokyo, 1981.

2. S. Akiyama, I. Matsumoto, N. Nakata, and R. Kaneko, Kobunshi Ronbunshu, 33, 238 (1976).

3. S. Kobayashi, S. Tazaka, and S. Miyata, Polym. Prepr. Jpn., 34, 1074 (1985).

4. R. Kaneko, H. Shimizu, and S. Akiyama, Kobunshi Kagaku, 23, 97 (1966).

5. P. J. Flory, "Principles of Polymer Chemistry," Cornell University Press, Ithaca, N. Y., 1953.

6. T. Nishi, J. Macromol. Sci., Phys. Ed., B17, 517 (1980).

7. R. E. Bernstein, C. A. Cruz, D. R. Paul, and J. W. Barlow, Macromolecules, 10, 681 (1977).

8. S. Akiyama and Y. Fujita, Polym. Prepr. Jpn., 36, 1197 (1978).

9. J. M. Barton, Polymer, 10, 151 (1969).

10. S. Akiyama, Y. Komatsu, and R. Kaneko, Polym. J., 7, 172 (1975)

11. S. Akiyama, J. Adhesion Soc. Jpn., 20, 485 (1984).

12. S. Akiyama, Shikizai Kyokaishi, 55, 165 (1982). 